\title{
ERRATA
}

\section{W-INFINITY WARD IDENTITIES AND CORRELATION FUNCTIONS IN THE $c=1$ MATRIX MODEL}

[Mod. Phys. Lett. A, Vol, 7, No. 11 (1992) 937-953] Sumit R. Das, Avinash Dhar, Gautam Mandal and Spenta R. Wadia

The affiliation of S. R. Wadia should be

Institule for Advanced Study, Olden Lane, Princeton NJ 08540, USA 